Paper presented at the IEEE Nuclear and Space

\title{
Investigation of the Oxygen-Vacancy (A-Center) Defect

\section{Complex Profile in Neutron Irradiated High Resistivity \\ Silicon Junction Particle Detectors*}

\author{
Zheng $\mathrm{Li}$ and $\mathrm{H}$. W. Kraner \\ Brookhaven National Laboratory, Upton, New York, USA \\ E. Verbitskaya, V. Eremin, and A. Ivanov \\ A. F. IOFFE Physico-Technical Institute \\ P.O. 194021, St. Petersburg, Russia \\ Monica Rattaggi and P. G. Rancoita \\ INFN, Milan, Italy \\ F. A. Rubinelli, ${ }^{\dagger}$ and S. J. Fonash \\ Center for Electronic Materials and Processing \\ Pennsylvania State University \\ University Park, Pennsylvania 16802, USA \\ C. Dale and P. Marshall \\ Naval Research Laboratory, Washington, DC, USA
}

February 1992

*This research was supported by the U. S. Department of Energy:

Contract No. DE-AC02-76CH00016.

$\dagger$ Permanent Address: INTEC Universidad Nacional del Litoral, Güemes 3450 (3600), Santa Fe, Argentina 


\title{
Investigation of the Oxygen-Vacancy (A-Center) Defect Complex Profile in Neutron Irradiated High Resistivity Silicon Junction Particle Detectors*
}

\author{
Zheng $\mathrm{Li}$ and $\mathrm{H}$. W. Kraner \\ Brookhaven National Laboratory, Upton, New York, USA \\ E. Verbitskaya, V. Eremin, and A. Ivanov \\ A. F. IOFFE Physico-Technical Institute \\ P.O. 194021, St. Petersburg, Russia \\ BNL- -47550 \\ Monica Rattaggi and P. G. Rancoita \\ INFN, Milan, Italy \\ DE92 016952 \\ F. A. Rubinelli, ${ }^{\dagger}$ and S. J. Fonash \\ Center for Electronic Materials and Processing \\ Pennsylvania State University, University Park, Pennsylvania 16802, USA \\ C. Dale and P. Marshall \\ Naval Research Laboratory, Washington, DC, USA
}

\begin{abstract}
Distributions of the A-center (oxygen-vacancy) in neutron damaged silicon detectors have been studied using Deep Level Transient Spectroscopy. A-centers have been found to be nearly uniformly distributed in the silicon wafer depth for medium resistivity $(0.1-0.2 \mathrm{k} \Omega-\mathrm{cm})$ silicon detectors. A positive filling pulse was needed to detect the A-centers in high resistivity $(>4 \mathrm{k} \Omega-\mathrm{cm})$ silicon detectors, and this effect was found to be dependent on the oxidation temperature. A discussion of this effect is presented.
\end{abstract}

\section{DISCLAIMER}

This report was prepared as an account of work sponsored by an agency of the United States Government. Neither the United States Government nor any agency thereof, nor any of their employees, makes any warranty, express or implied, or assumes any legal liability or responsibility for the accuracy, completeness, or usefulness of any information, apparatus, product, or process disclosed, or represents that its use would not infringe privately owned rights. Reference herein to any specific commercial product, process, or service by trade name, trademark, manufacturer, or otherwise does not necessarily constitute or imply its endorsement, recommendation, or favoring by the Unired States Government or any agency thereof. The views and opinions of authors expressed herein do not necessarily state ur reflect those of the United States Government or any agency thereof. 


\section{SUMMARY}

Silicon junction particle detectors manufactured on high resistivity n-type silicon wafers ( $\rho \geq 4 \mathrm{k} \Omega$-cm or $\mathrm{N}_{\mathrm{d}}<10^{12} / \mathrm{cm}^{3}$ ) have been widely used in high energy physics experiments. Silicon detector systems proposed for the new large accelerators, the Superconducting Super Collider and the Large Hadron Collider, will be subject to significant radiation damage from both ionizing and fast neutron fluences. One of the major concerns is the effect of neutron radiation damage causing leakage current degradation [1-4], dopant compensation and/or donor removal [2], and type-inversion ( $\rightarrow$ p) [5]. It may be possible to minimize the bulk displacement damage of fast neutrons by controlling the initial impurities in the silicon crystal. Oxygen, for example, has been used [6-7] to "getter" vacancies (V) through the formation of the A-center $\left(\mathrm{O}-\mathrm{V}, \mathrm{E}_{\mathrm{c}}-0.18 \mathrm{eV}\right)$, and because of its competition for the damage-induced vacancies, decrease the concentration of the deeper $\mathrm{E}-$ Center $\left(\mathrm{P}-\mathrm{V}, \mathrm{E}_{\mathrm{c}}-0.44 \mathrm{eV}\right)$ and the divancy, which are much more effective SRH gneration centers than A-center. Thus, gettering of vacancies might help to decrease the leakage current degradation and donor removal. A detailed study of the A-center as a function of material resistivity and processing schedules was undertaken to help understand its availablity and effectiveness as such a getter. The determination of the profile of the A-center in the depth of the silicon junction detector fabricated with oxides formed at several oxidation temperatures may help resolve issues concerning of type-inversion at high neutron fluences $\left(>10^{13} \mathrm{n} / \mathrm{cm}^{2}\right)$ where there appears to be a difference between the surface region and the bulk [7-8] and a less understood role between the A-center and thermal donors. Further, basic information on defect characteristics of high resistivity material will be important to suggest other material hardening approaches should gettering by $A$-centers prove not to be entirely $;$ orthwhile.

Deep Level Transient Spectroscopy (DLTS) has been widely used to characterize the parameters (e.g., the trap energy $E_{t}$, its crossection $\sigma_{t}$, and its concentration $N_{t}$ ) of single defects in low resistivity silicon $(\rho<10 \Omega-\mathrm{cm})$ caused by electrons, $\gamma$-ray [9-10] and neutrons [11-12]. Some DLTS and thermally stimulated current (TSC) work on high resistivity silicon detectors has been reported [13-14], but does not deal with the profile of A-centers versus depth into the detector or the effects of processing thereon. In this work, A-centers in neutron damaged detectors fabricated on various oxides $\left(975^{\circ}-1200^{\circ} \mathrm{C}\right.$ in oxygen ambient with and without TCA, trichloroethane) are studied using DLTS, and the profile of A-centers in medium resistivity silicon $(\rho \sim 0.1$ to $0.2 \mathrm{k} \Omega-\mathrm{cm}$ ) is determined. The junction detectors used in this study were implanted $\mathrm{p}^{+}-\mathrm{n}-\mathrm{n}^{+}$detectors made on $\mathrm{n}$-type $\langle 111\rangle$ silicon wafers, with intermediate resistivities of $0.15 \mathrm{k} \Omega$-cm in order to directly compare float zone (lower as-grown oxygen) and Czochralski (high as-grown oxygen, available only up to this resistivity) and representative, high resistivity, detector grade, float zone material of $4 \mathrm{k} \Omega-\mathrm{cm}$. Different oxides, with oxidation temperature ranging from $975^{\circ} \mathrm{C}$ to $1200^{\circ} \mathrm{C}$, were used to introduce various concentrations of oxygen [6], into the high resistivity $\mathrm{FZ}$ silicon. Fast neutrons from $10 \mathrm{keV}$ to $2.2 \mathrm{MeV}$, with $\overline{\mathrm{E}}=1 \mathrm{MeV}$, were obtained from the ${ }^{7} \mathrm{Li}(\mathrm{p}, \mathrm{n})$ reaction using $4 \mathrm{MeV}$ protons.

Table I lists the A-center concentration determined by DLTS measurements on neutron damaged detectors made on medium resistivity $\mathrm{CZ}$ and $\mathrm{FZ}$ silicon. In spite of the vast difference of oxygen concentrations $\left(\mathrm{N}_{\mathrm{o}}\right)$ between $\mathrm{CZ}$ silicon $\left(\mathrm{N}_{\mathrm{o}}-10^{17} / \mathrm{cm}^{3}\right)$ and $\mathrm{FZ}$ silicon $\left(N_{0}-10^{15} / \mathrm{cm}^{3}\right)$, the A-center concentrations are almost the same for the two materials damaged to about $5 \times 10^{11} \mathrm{n} / \mathrm{cm}^{2}$. Our data also have shown no difference in leakage current for 
detectors damaged up to a few times $10^{12} \mathrm{n} / \mathrm{cm}^{2}$, indicating no gettering effect in the low neutron fluence region ( $\left.\leq 10^{12} \mathrm{n} / \mathrm{cm}^{2}\right)$ [7].

Table I. DLTS data of A-center concentrations for $n$-damaged $\mathrm{CZ}$ and FZ silicon detectors $\phi_{\mathrm{n}}=5.0 \times 10^{11} \mathrm{n} / \mathrm{cm}^{2}$

\begin{tabular}{|c|c|c|c|c|}
\hline & $\begin{array}{r}\# 138-7 \\
0.2 \mathrm{k} \Omega-\mathrm{cm}\end{array}$ & $\begin{array}{l}\text { CZ Silicon } \\
1100^{\circ} \mathrm{C} \text { oxide }\end{array}$ & $\begin{array}{r}\# 141-3 \\
0.2 \mathrm{k} \Omega-\mathrm{cm} \\
\end{array}$ & $\begin{array}{l}\text { FZ Silicon } \\
1100^{\circ} \mathrm{C} \text { oxide }\end{array}$ \\
\hline A-Center Content & $3.0 \times$ & $10^{11} / \mathrm{cm}^{3}$ & $3.1 \times$ & $10^{11} / \mathrm{cm}^{3}$ \\
\hline
\end{tabular}

Physical parameters of the A-center, E-center and double vacancy $(\mathrm{V}-\mathrm{V})$ centers in different charge states for a medium resistivity FZ silicon detector are shown in Table II. These results essentially agree with other measurements of these defects $[9,10,11,12,13]$.

Table II. Trap parameters for a FZ silicon detector (\#143-7), $\phi_{\mathrm{n}}=5 \times 10^{11}$ $\mathrm{n} / \mathrm{cm}^{2}, \rho=0.2 \mathrm{k} \Omega-\mathrm{cm}, 1100^{\circ} \mathrm{C}$ oxide.

\begin{tabular}{cccc}
\hline & A-Center & $\mathrm{V}-\mathrm{V}^{=}$ & E-Center and $\mathrm{V}-\mathrm{V}^{-}$ \\
\hline $\mathrm{E}_{\mathrm{c}}-\mathrm{E}_{\mathrm{t}}(\mathrm{eV})$ & 0.17 & 0.21 & 0.41 \\
$\sigma_{\mathrm{t}}\left(\mathrm{cm}^{2}\right)$ & $2 \times 10^{-15}$ & $1.3 \times 10^{-16}$ & $6 \times 10^{-16}$ \\
$\mathrm{~N}_{\mathrm{t}}\left(\mathrm{cm}^{-3}\right)$ & $2.5 \times 10^{11}$ & $1.2 \times 10^{11}$ & $3.3 \times 10^{11}$ \\
\hline
\end{tabular}

Figure 1 shows the A-center concentration as a function of depletion depth obtained by DLTS measurements on detector \#143-7 (FZ, medium resistivity). This profile was obtained by varying the depletion voltage while keeping the difference between the pulse maximum $\left|V_{\max }\right|$ and minimum $\left|V_{\min }\right|$ at a constant, i.e., $\left|V_{\max }\right|-\left|V_{\min }\right|=1$ Volt. One can therefore study those traps near the depletion edge [15]. A specific distribution of the A-center cannot be distinguished as either a near-surface or uniform through the bulk distribution from the range of depletion depths available from material of this resistivity.

For high resistivity silicon $(4 \mathrm{~K} \Omega-\mathrm{cm})$, however, it has been found that one has to use a positive filling pulse (forward bias, $0<\mathrm{V}<0.7 \mathrm{~V}$ ) to detect the A-center concentration. Table III summarizes the data of the dependence of the A-center concentration on the positive filling bias for detectors fabricated after various temperature/oxidation schedules. The negative results for the high temperature oxidation (first column) are included only for direct comparison with the other samples as they are results from the search as a function of filling bias. The positive filling bias effect becomes more evident as the oxidation temperature increases. This effect does not occur in medium resistivity silicon detectors regardless of oxidation temperature; the Acenter was observable without requiring the fill pulse to go positive. No significant differences in E-center concentrations among detectors made on various oxides up to $\phi_{n}=5 \times 10^{11} \mathrm{~cm}^{2}$ were observed. 


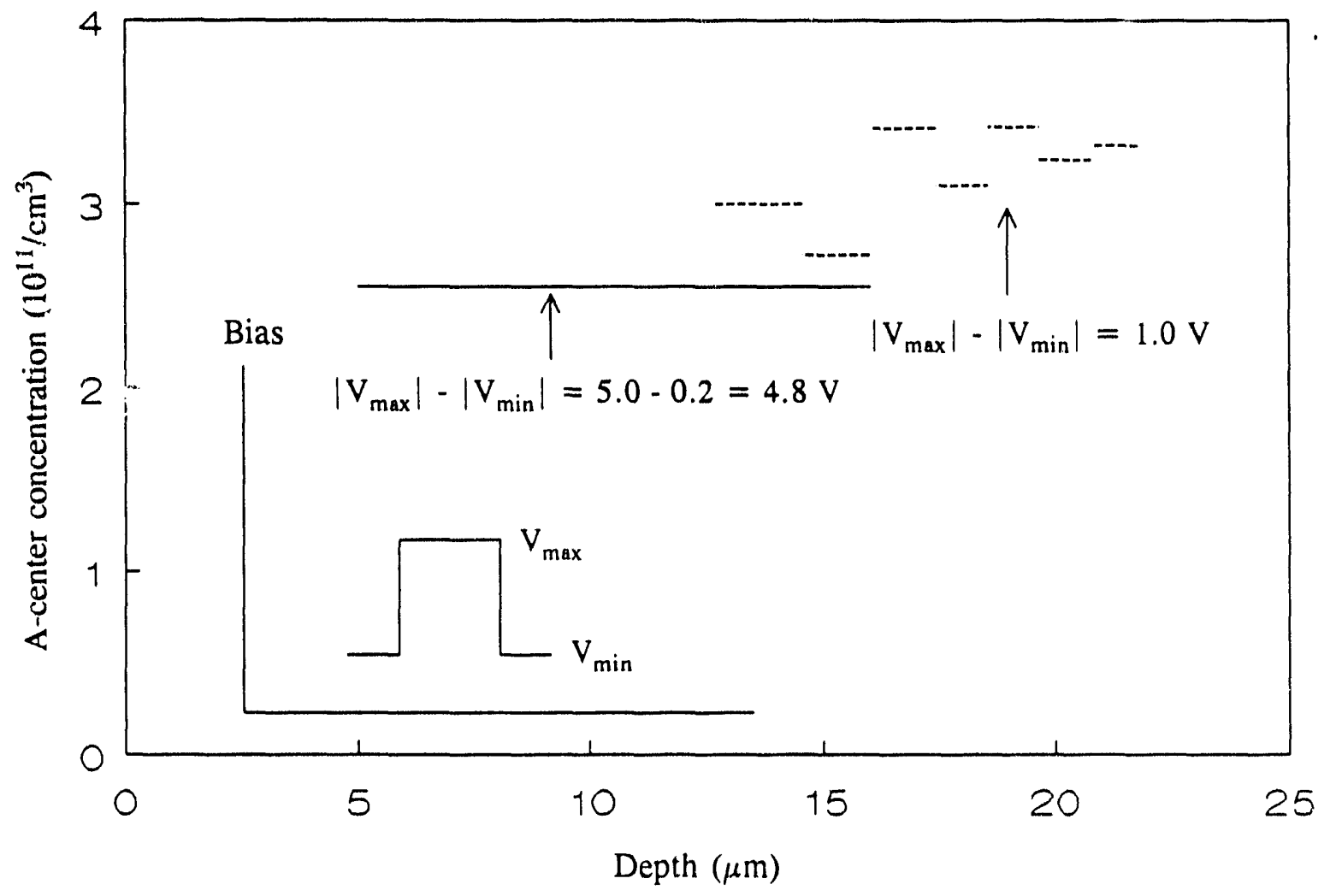

Fig. 1. A-center profile obtained from DLTS using various reverse bias with fixed $\left|\mathrm{V}_{\max }\right|-\left|\mathrm{V}_{\min }\right| . \quad\left(\# 143-7, \rho=0.1-0.2 \mathrm{k} \Omega-\mathrm{cm}, \phi_{\mathrm{n}}=5 \times 10^{11} \mathrm{n} / \mathrm{cm}^{2}\right)$ The solid line extending to $15 \mu \mathrm{m}$ is the concentration for the full bias of $5 \mathrm{~V}$ including the volume up to the depletion of $15 \mu \mathrm{m}$.

The difficulty in observing the A-center in high resistivity samples compared with the medium resisitivity samples must lie in part with the different positions of the fermi level, which lies below the A-center in the $4 \mathrm{~K} \Omega$ material, but very close to the A-center defect level at medium resisitivity. Therefore substantial fast detrapping must occur for the majority carriers after filling under normal conditions with large reverse biases. Slight forward biases, however, permit filling of centers near the surface, $p+$ contact, where band bending permits the centers to remain filled longer over the course of the measurement. A slight positive bias, of the order of the diffusion potential is required to do this.

Table III. A-center concentrations at various filling voltages, $\phi_{\mathrm{n}}=5.0 \times 10^{11} \mathrm{n} / \mathrm{cm}^{2}$ 


\begin{tabular}{cccc}
\hline & $\begin{array}{c}\text { \#132-3 FZ } 4 \mathrm{k} \Omega \text {-cm } \\
1200^{\circ} \mathrm{C} \text { Oxide+TCA }\end{array}$ & $\begin{array}{c}\# 130-4 \mathrm{FZ} 4 \mathrm{k} \Omega-\mathrm{cm} \\
1100^{\circ} \mathrm{C} \mathrm{Oxide}\end{array}$ & $\begin{array}{c}\# 133-4 \mathrm{FZ} 4 \mathrm{k} \Omega \text {-cm } \\
975^{\circ} \mathrm{C} \text { Oxide }\end{array}$ \\
\hline $\mathrm{V}_{\text {fill }}(\mathrm{V})$ & A-center $\left(\mathrm{cm}^{-3}\right)$ & A-center $\left(\mathrm{cm}^{-3}\right)$ & $\mathrm{A}$-center $\left(\mathrm{cm}^{-3}\right)$ \\
\hline 1.0 & not detected & $7.2 \times 10^{10}$ & $1.3 \times 10^{11}$ \\
0.9 & not detected & $1.8 \times 10^{11}$ & $1.6 \times 10^{11}$ \\
0.8 & not detected & $4.2 \times 10^{11}$ & $3.0 \times 10^{11}$ \\
0.7 & not detected & $1.10 \times 10^{11}$ & $6.5 \times 10^{11}$ \\
0.6 & not detected & $1.0 \times 10^{11}$ & $6.0 \times 10^{11}$ \\
0.5 & not detected & $7.2 \times 10^{10}$ & $6.0 \times 10^{11}$ \\
0.4 & not detected & $6.3 \times 10^{10}$ & $6.1 \times 10^{11}$ \\
0.3 & not detected & $3.1 \times 10^{10}$ & $5.7 \times 10^{11}$ \\
0.2 & not detected & $<10^{10}$ & $5.7 \times 10^{11}$ \\
0.1 & not detected & & $4.6 \times 10^{11}$ \\
0.0 & not detected & $<10^{10}$ & $2.9 \times 10^{11}$ \\
-0.5 & not detected & & $4.5 \times 10^{10}$ \\
-1.0 & not detected & $<10^{10}$ & $4.5 \times 10^{10}$ \\
-1.5 & not detected & & $4.8 \times 10^{10}$ \\
-2.0 & not detected & & $4.8 \times 10^{10}$ \\
-3.5 & not detected & & $5.6 \times 10^{10}$ \\
-5.0 & not detected & & $5.5 \times 10^{10}$ \\
\hline
\end{tabular}

(Some data for a $1200 \mathrm{C}$ oxidation without TCA did yield a detectable A-center concentration of about $5 \times 10^{11} / \mathrm{cm}^{3}$ only for filling pulses between +0.3 and +0.8 volts).

If one pursues the possibility of the A-centers being surface concentrated, one can consider the oxidation conditions. The $1200 \mathrm{C}$ oxidation parameters were chosen to follow [6] who after reasonable assumptions about the source oxygen concentration believe one achieves a concentration of at least $10^{17} / \mathrm{cm}^{2}$, to be equivalent to $\mathrm{CZ}$ material, throughout the bulk of the wafer with an approximate diffusion length of $60 \mu \mathrm{m}$. The lower temperature oxidations give diffusion lengths of a few microns. If one assumes the source oxygen concentration--at the surface--is essentially equal for both high and low temperature processes, one would not expect a substantially different near-surface oxygen concentration acting as a sink for vacancies and thus giving the higher A-center concentration observed. The shorter time lower temperature oxidations might, however, produce a higher concentration of free, available, uncomplexed oxygen for more effective A-center formation. Alternatively, the high temperature (1200C) oxidation using oxygen plus TCA is known to create a denuded zone of silicon interstitials near the surface and some growth of interstitial precipitates in the bulk during cool-down[16]. The silicon interstitials and precitates, though still a good sink for vacancies, may not provide enough free oxygen to form single defect A-centers. Certainly, further experiments with samples having different thermal histories may help confirm these suggestions.

We have shown that the A-center has a nearly uniform depth profile for medium resistivity silicon detectors under a variety of oxidation conditions after somewhat low fast neutron fluences. In high resisitivity silicon, the detection of the A-center is dependent on both 
the fill pulse and the oxidation process. Measurements will continue to further explore A-center behavior and to quantify the depth profile in high resistivity silicon.

\section{References}

[1] H. W. Kraner, "Radiation Damage in Silicon Detectors," Nucl. Instrum. Methods 225, 615-618, (1984).

[2] H. Dietl, T. Gooch, D. Kelsey, R. Klanner, A. Loffler, M. Pepe, and F. Wickens, "Radiation Damage in Silicon Strip Detectors," Nucl. Instrum. Methods A253, 460-466, (1987).

[3] H. W. Kraner, Z. Li, and K. V. Pösnecker, "Fast Neutron Damage in Silicon Detectors," Nucl. Instrum. Methods A279, 266-271, (1989).

[4] G. Lindström, E. Fretwurst, H. Herdan, M. Rollwagen, P. Thomsen, and R. Wunstorf, "Radiation Damage Effects in Silicon Detectors," DESY 89-105, August (1989).

[5] G. Lindström, M. Benkert, E. Fretwurst, T. Schulz and R. Wunsdorf, "Radiation Effects in Si Detectors and Calorimeter Considerations," Proc. of the First International Conf. on Calorimetry in High Energy Physics, D. F. Anderson, et al., eds., (World Scientific Publishing Co., Singapore, 1991).

[6] S. D. Brotherton and P. Bradley, "Defect Production and Lifetime Control in Electron and $\gamma$ irradiated Silicon," J. Appl. Phys. 53(8), 5720 (1982).

[7] Zheng Li and H. W. Kraner, "Studies of the Dependence on Oxidation Thermal Processes of Effects on the Electrical Properties of Silicon Detectors by Fast Neutron Radiation," presented at the 1991 IEEE Nucl. Sci. Symp., Santa Fe, NM, November 5-9, 1991.

[8] H. W. Kraner, E. Fretwurst, and Zheng Li, "Determination of Internal Electric Field Profile in Neutron Damaged Silicon Detectors Using Current Pulse Shape," to be presented at the Sixth European Symp. on Semiconductor Detectors, Milan, Italy, February 24-26, 1992.

[9] L. C. Kimerling, H. M. DeAngelis and J. W. Diebold, "On the Role of Defect Charge State in the Stability of Point Defects in Silicon," Solid State Comm. 16, 171-174 (1975).

[10] L. C. Kimerling, "Defect States in Electron-bombarded Silicon: Capacitance Transient Analyses, " in: Radiation Effects in Semiconductors, (The Institute of Physics, Bristol, UK), 1976 Institute of Physics Conf. Series 31, 221-230 (1977).

[11] Y. Tokuda and A. Usami, "Comparison of Neutron and $2 \mathrm{MeV}$ Electron Damage in n-type Silicon by Deep-level Transient Spectroscopy," IEEE Trans. Nucl. Sci. NS-28(3), 3564-3568 (1981).

[12] Y. Tokuda and A. Usami, "DLTS Studies of Neutron Damage in p-type Silicon," IEEE Trans. Nucl. Sci. NS-29(5), 1388-1392 (1982).

[13] E. Borchi, C. Bertrand, C. Leroy, M. Bruzzi, C. Furetta, R. Paluletto, P. G. Rancoita, L. Vismara, and P. Giubellino, "Deep-level Transient Spectroscopy Measurements of Majoriry Carrier Traps in Neutron-Irradiated n-type Silicon Detectors," Nucl. Instrum. Methods, $\underline{\mathbf{A 2 7 9}}$. 277-280 (1989).

[14] Zheng Li, W. Chen and H. W. Kraner, "Effects of Fast Neutron Radiation on the Electrical Properties of Silicon Detectors," Nucl. Instrum. Methods A308, 585-595 (1991).

[15] V. K. Eremin, A. M. Ivanov, N. B. Strokan and V. Shokina, Sov. Phys. Semicond. 23(9), 998 (1989).

[16] Zheng Li and H. W. Kraner, "Gettering in High Resistivity Float-Zone Silicon Wafers or Silicon Detector Applications", IEEE Trans. Nucl. Sci., NS-37(1) 290 (1989). 

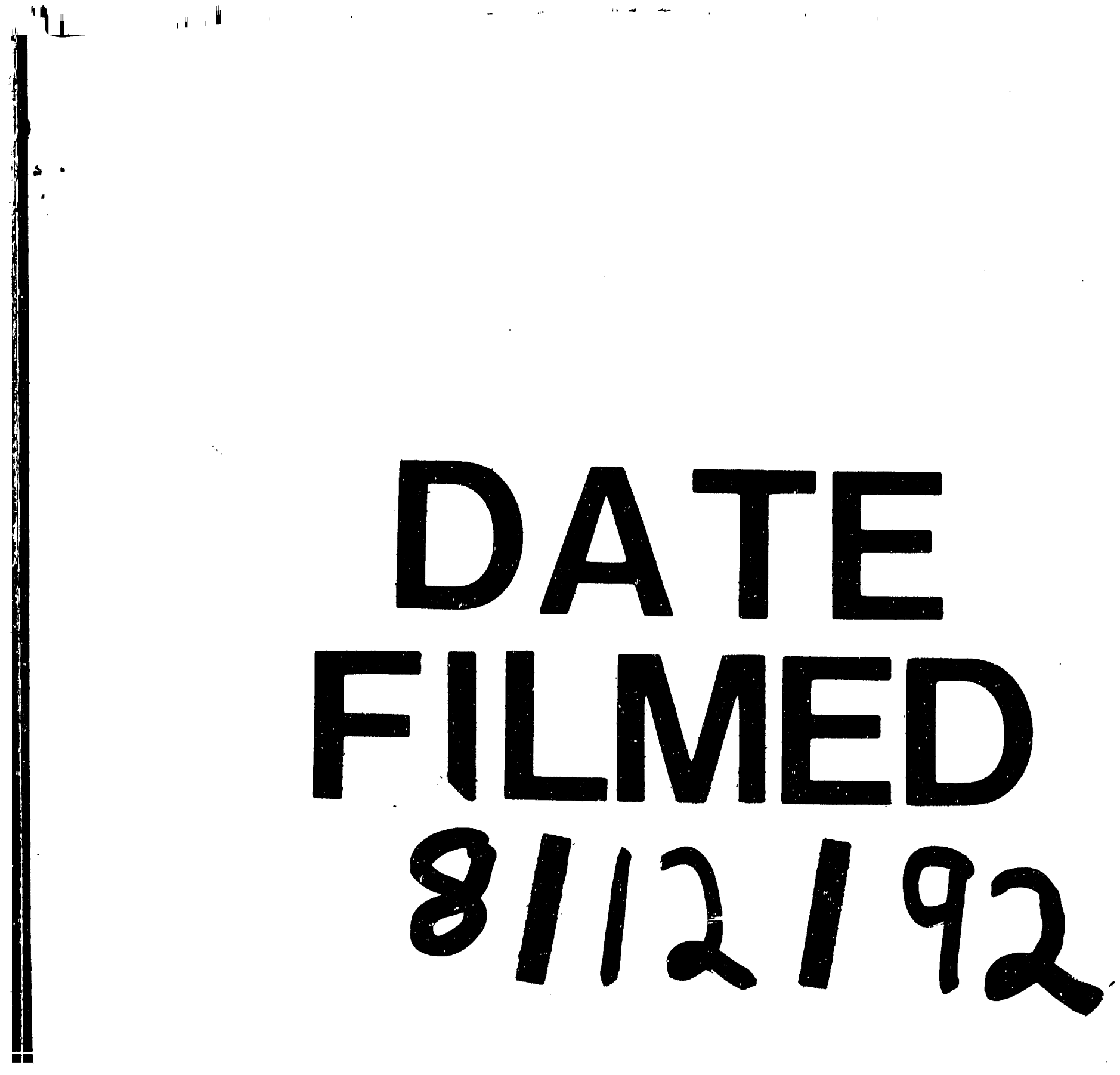


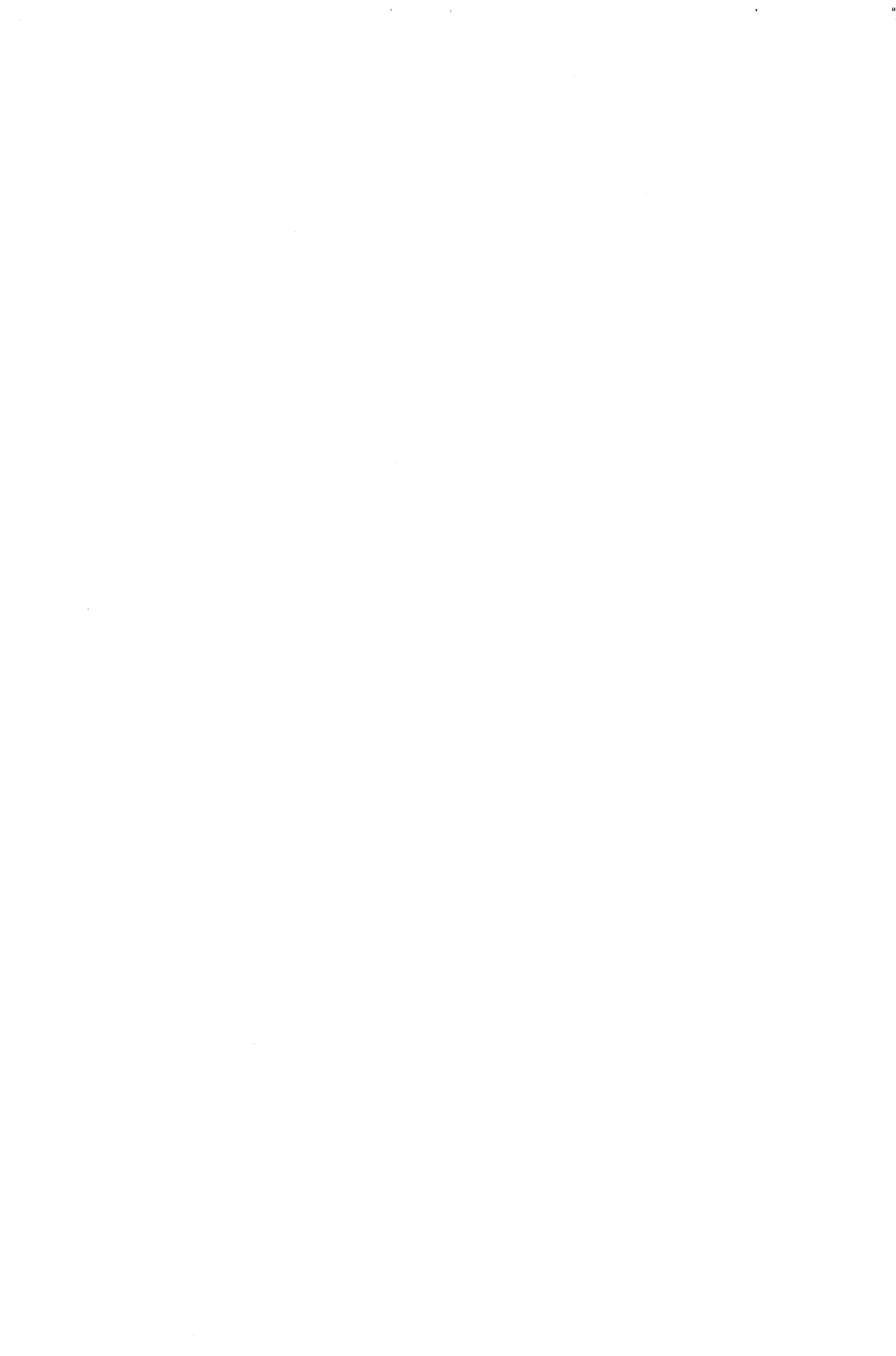

